\title{
Theoretical verification of the backward displacement of waves reflected from an interface having superimposed periodicity
}

\author{
Nico F. Declercq and Joris Degrieck \\ Soete Laboratory, Department of Mechanical Construction and Production, Ghent University, Sint \\ Pietersnieuwstraat 41, 9000 Gent, Belgium. \\ Rudy Briers \\ KATHO (RENO department), Sint Jozefstraat 1, 8820 Torhout, Belgium \\ Oswald Leroy \\ Interdisciplinary Research Center, Katholieke Universiteit Leuven Campus Kortrijk, E. Sabbelaan 53, \\ 8500 Kortrijk, Belgium
}

(Received 21 October 2002; accepted 14 February 2003)

\begin{abstract}
Experiments are reported by Breazeale and Torbett [Appl. Phys. Lett. 29, 456 (1976)] that visualize ultrasonic backward beam displacement due to the excitation of surface waves by means of diffraction. The authors have simulated these experiments using the concept of inhomogeneous waves. Such waves have proven to be well suited in predicting beam displacements on plane interfaces. It is now found that inhomogeneous waves are even capable of predicting the experimentally observed displacement phenomena on periodic rough surfaces. (C) 2003 American Institute of Physics. [DOI: 10.1063/1.1567043]
\end{abstract}

Based on the optical theory, it was predicted by Bertoni and Tamir, ${ }^{1}$ by means of interpreting the classical grating equation for a periodic structure, that the coupling of incident light with surface waves on periodically corrugated surfaces can cause not only a forward beam displacement, but also a backward beam displacement. Therefore, Breazeale et al. ${ }^{2}$ decided to find out experimentally if this analogy also existed in the acoustic wave theory. They showed that a wave having an angle of incidence $\theta_{i}$ generates a backward propagating surface wave if the following relation holds:

$$
\sin \theta_{i}=v_{i}\left(\frac{1}{f \Lambda}-\frac{1}{v_{\sigma}}\right),
$$

where $v_{i}$ is the sound velocity in the incidence medium, $v_{\sigma}$ is the velocity of the surface wave, $\Lambda$ is the period of the grating, and $f$ is the sound frequency. Breazeale et al., ${ }^{2}$ verified that on a water/brass interface, having a superimposed periodicity $\Lambda=0.178 \mathrm{~mm}$ and a corrugation depth $t$ $=0.025 \mathrm{~mm}$, a backward displacement occurs (see Fig. 1) if a bounded beam is used of a physical width of approximately $10 \mathrm{~mm}$, a frequency of $6 \mathrm{MHz}$, and incidence angle $\theta_{i}$ $=22.5^{\circ}$. They also found that the phenomenon disappears if a frequency of $2 \mathrm{MHz}$ is used under the same angle of incidence. They calculated that the velocity of the surface wave that causes this effect of a backward beam shift is approximately $1470 \mathrm{~m} / \mathrm{s}$.

An inhomogeneous wave is mathematically described as a well known pure infinite plane wave, except that its wave vector is complex valued. Physically, this results in exponential decay of its amplitude along the wave front. The theory of the diffraction of inhomogeneous waves, ${ }^{3}$ which is applied here, consists of the decomposition of the diffracted wave fields into inhomogeneous waves having an amplitude that is determined by the continuity of normal stress and displace- ment and having a propagation direction and inhomogeneity that is determined by an extension ${ }^{3}$ of the classical diffraction equation. ${ }^{4}$ In that diffraction theory, we have incorporated the findings of Deschamps, ${ }^{5}$ as to determine the correct direction of all excited waves. Furthermore, the incident bounded beam is theoretically built up by inhomogeneous waves as described in literature. ${ }^{6,7}$

Figure 2 recalls the experiments of Breazeale $e t$ al. ${ }^{2}$ In Figs. 3 and 4, the simulations are shown under the same circumstances as in Fig. 2. Breazeale et al. ${ }^{2}$ noted that they could not observe this phenomenon if in Eq. (1) the parameters for a backward traveling leaky Rayleigh wave are entered since this corresponds to an incidence angle of $41^{\circ}$ where experimentally nothing out of the ordinary occurs. The actual nature of the phenomenon at $\theta_{i}=22.5^{\circ}$ remained obscure.

The fact that the concept of inhomogeneous waves enables us to simulate such strange effects on rough surfaces is yet another verification of its usefulness, as had already been noted in simulations ${ }^{6,7}$ of beam displacements on smooth

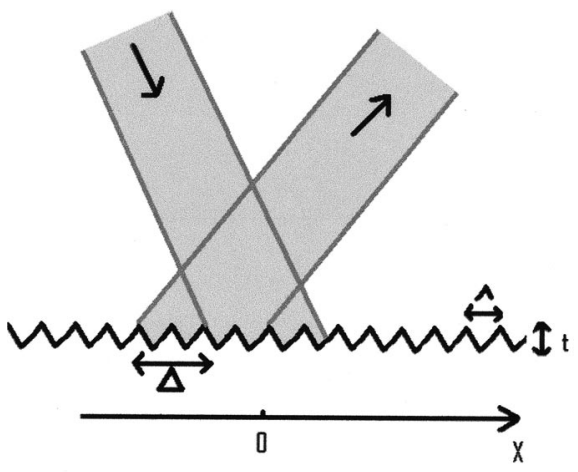

FIG. 1. Diagram of the backward beam displacement on a corrugated surface. 


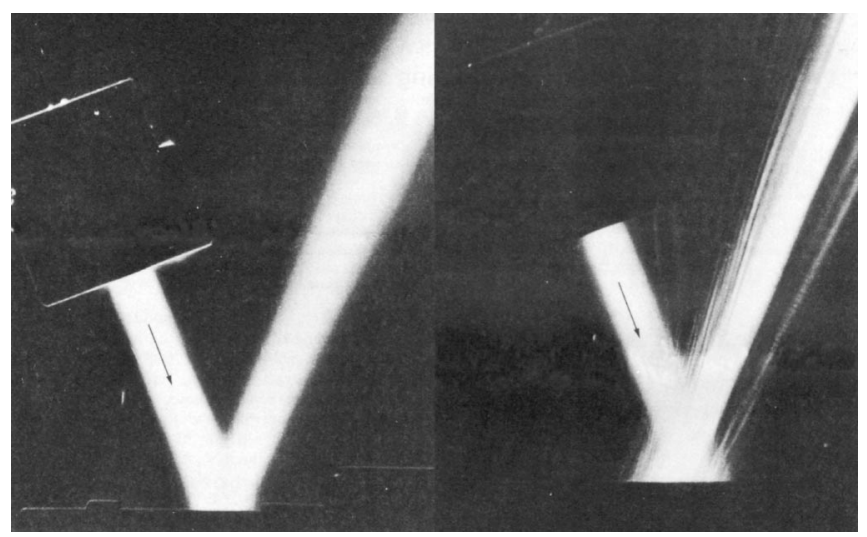

FIG. 2. Diffraction experiments of Breazeale and Torbett (as reported in Ref. 2). The experiments were performed at $\theta_{i}=22.5^{\circ}$. The left-hand side shows the case for $2 \mathrm{MHz}$, while the right-hand side depicts the situation at $6 \mathrm{MHz}$.

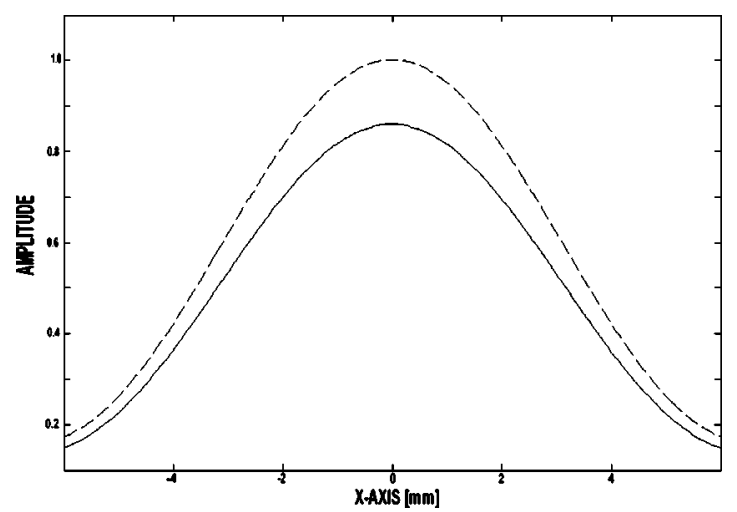

FIG. 3. Dashed line: Incident Gaussian beam profile as a superposition of inhomogeneous waves. Full line: Calculated reflected beam profile as a superposition of the reflected inhomogeneous waves by which the incident field is built up. $x$ axis in [m]. Frequency: $2 \mathrm{MHz}$, angle of incidence: $22.5^{\circ}$. No displacement is observed.



FIG. 4. The same curves as in Fig. 3, but for 6 MHz. Clearly, a backward beam displacement appears over a distance $\Delta$.

surfaces. ${ }^{8}$ However, even though it is reported here that the diffraction of inhomogeneous waves predicts what Breazeale et $a .^{2}$ have observed experimentally, coming to a final conclusion as to what actually causes the effect remains a subject for further theoretical research.

The authors are grateful to "The Flemish Institute for the Encouragement of the Scientific and Technological Research in Industry (I.W.T.)" for financial support.

${ }^{1}$ T. Tamir and H. L. Bertoni, J. Opt. Soc. Am. 61, 1397 (1971).

${ }^{2}$ M. A. Breazeale and M. A. Torbett, Appl. Phys. Lett. 29, 456 (1976).

${ }^{3}$ R. Briers, O. Leroy, O. Poncelet, and M. Deschamps, J. Acoust. Soc. Am. 106, 682 (1999).

${ }^{4}$ N. F. Declercq, R. Briers, J. Degrieck, and O. Leroy, IEEE Trans. Ultrason. Ferroelectr. Freq. Control 49, 1516 (2002).

${ }^{5}$ M. Deschamps, J. Acoust. Soc. Am. 96, 2841 (1994).

${ }^{6}$ J. M. Claeys and O. Leroy, J. Acoust. Soc. Am. 72, 585 (1982).

${ }^{7}$ K. Van Den Abeele and O. Leroy, J. Acoust. Soc. Am. 93, 308 (1993).

${ }^{8}$ M. A. Breazeale, L. Adler, and G. W. Scott, J. Appl. Phys. 48, 530 (1977). 
Applied Physics Letters is copyrighted by the American Institute of Physics (AIP). Redistribution of journal material is subject to the AIP online journal license and/or AIP copyright. For more information, see http:/ojps.aip.org/aplo/aplcr.jsp

Copyright of Applied Physics Letters is the property of American Institute of Physics and its content may not be copied or emailed to multiple sites or posted to a listserv without the copyright holder's express written permission. However, users may print, download, or email articles for individual use. 